\title{
Is It The Rock or The Frac? Shale Reservoir Quality and Production Performance
}

Author: Randy Miller (Core Lab)

Post frac production performance from source rock reservoirs varies significantly between various formations, regionally within a shale formation, and even within a field area. Are these variances caused by differences in reservoir quality, stimulation efficiencies, or both? In order to understand this problem and manage well performance expectations, it is essential to determine the key reservoir quality parameters that correlate with production performance, and be able to evaluate the stimulation efficiency. By benchmarking reservoir quality to production performance, underperforming wells can be identified and the stimulation can then be optimized. This is best accomplished by evaluating a number of cored vertical pilot wells and their corresponding laterals.

Source rock reservoir quality is influenced by a number of parameters. These have been reported in the literature to include TOC, geochemical maturity, porosity, matrix and system permeability, fractures, mineralogy, brittleness, thickness, reservoir pressure, vertical and lateral heterogeneity, and closure stress. All of these parameters may have an impact on reservoir quality, however, in each shale play a few of the parameters dominate significantly over the others as drivers of production. The goal is to then determine which ones are the drivers in the play.

The goal of the completion engineer is to optimize the fracture stimulation in terms of production rate and ultimate recovery at an economic cost. The challenge for the completion engineer is that there many parameters to optimize. These include lateral direction, lateral length, lateral landing point, toe up or toe down, number of stages, stage spacing, cluster spacing, perforation orientation, fluid systems, proppant type, fluid volumes, proppant volumes, pump rates, and more. In order to properly evaluate the stimulation, the engineer has a number of post-frac tools that are available. These include proppant tracers, chemical flow-back tracers, production logging, micro-seismic monitoring, and production analysis. The use of these technologies allows the engineer to determine if proppant went into all the perforation clusters, flow-back efficiency of the various stages, which stages are contributing to production and which may not be, fracture height growth, effective frac length, drainage area, and the producing permeability.

A case history demonstrates that reservoir quality is the primary driver to production performance and that optimizing the fracture stimulation treatment has a secondary impact. The concept of the Shale Reservoir Quality Index (SRQI) is introduced and it is shown to correlate well with cumulative production. The resulting model also allows the identification of poorly stimulated wells. 